\title{
The Application of Adaptive LMF Quadratic and Cubic Volterra Filters to ECG Signals
}

\author{
W. A. Zgallai
}

\begin{abstract}
This paper proposes to decompose the maternal transabdominal ECG signal into its linear, quadratic and cubic parts and retain only the linear part for further signal processing. The synthesiser will model the quadratic and cubic parts of the transabdominal ECG signal. This is done by predicting each sample of the maternal transabdominal full cardiac cycles. In order to linearise (retain the linear part and remove the quadratic and cubic parts) ECG signals, non-linear structures should be utilised. This paper proposes to linearise the fetal scalp electrode, the maternal chest, and the maternal transabdominal ECG signals using adaptive least-mean-square (LMS) and least-mean-fourth-based (LMF), quadratic and cubic Volterra structures.
\end{abstract}

Index Terms-Adaptive algorithms, volterra filters, ECG signals.

\section{INTRODUCTION}

The maternal transabdominal ECG signal is non-Gaussian and possesses quadratic and cubic non-linearities [1]. The contaminating noise contains Gaussian, non-Gaussian, and non-linear components [2]. The maternal transabdominal ECG signal is a combined maternal and fetal ECG and there is also another signal combined with it, the uterine contraction interference signal during labour [3]. Each one of these combined signals is non-linear by its own right [4]. A non-linear synthesiser is sought to carry out this task because employing a linear structure to cater for such non-linear signals would lead to a suboptimal solution.

The structure of the paper is as follows; First brief summaries of the standard LMS and LMF algorithms are given in Section II. This is followed by descriptions of the LMS- and LMF- Quadratic and Cubic Volterra structures in Section III. The Volterra synthesisers are applied to fetal scalp electrode, maternal chest, and maternal transabdominal ECG signals in Section 4. Conclusions are drawn in Section $\mathrm{V}$. The following abbreviations are followed; LMSQV: LMS-Quadratic Volterra, LMFQV: LMF-Quadratic Volterra, LMSCV: LMS-Cubic Volterra, and LMFCV: LMF-Cubic Volterra.

\section{ADAPTIVE LMS AND LMF ALGORITHMS}

Filtering implies extracting information from a signal at time $t$ by using data before and including time $t$. Prediction, however, is aimed at deriving information about the signal at time $t+\Delta$ in the future by using data available before and

Manuscript received June 10, 2014; revised August 8, 2014

W. A. Zgallai is with the Faculty of Engineering Technology and Science, Higher Colleges of Technology, UAE (e-mail: walidzgallai@yahoo.co.uk). including time $t$. The design of Wiener filters, which is a class of optimum linear discrete-time filters, requires prior information about the statistics of the data to be processed. However, adaptive filters do not require such information. For adaptive filters, the parameters of the filter are updated from one iteration to the next. The parameters become data dependent which makes it possible for the filter to perform satisfactorily in an environment where complete knowledge of the relevant signal statistics is not available. In a stationary environment, after successive iterations of the adaptive filtering algorithm it converges to the optimum Wiener solution in some statistical sense. In a non-stationary environment, the algorithm offers a tracking capability, whereby it can track time variations in the statistics of the input data, provided that the variations are sufficiently slow. Adaptive algorithms have fast speed of operation in terms of the CPU time and they are computationally efficient. There are four basic classes of adaptive filtering applications: (a) class I: identification; (b) class II: inverse modelling; (c) class III: prediction; and (d) class IV: interference cancellation.

The issue of prediction is one of the most basic and pervasive learning tasks. It is a temporal signal processing problem in that we are given a set of $N$ past samples $x(n-1)$, $x(n-2), \ldots, x(n-N)$ that are usually uniformly spaced in time, and the requirement is to predict the present sample $x(n)$. Prediction may be solved using error-correction learning in a supervised manner in the sense that the training examples are drawn directly from the time series itself. Specifically, the sample $x(n)$ serves the purpose of the desired response; hence given the corresponding prediction $x(n)$ produced by the structure on the basis of the previous samples $x(n-1), x(n-2)$, $\ldots, x(n-N)$, the prediction error may be computed as:

$$
e(n)=x(n)-\hat{x}(n \mid n-1, \ldots, n-N),
$$

and thus use the error-correction learning to modify the step-size parameter of the structure. Prediction may be viewed as a form of model building in the sense that the smaller we make the prediction error in a statistical sense, the better will the structure serve as a statistical model of the underlying process responsible for the generation of the time series. The problem of designing an optimum linear filter that provides the theoretical framework for linear adaptive filters was first conceived by Kolmogorov [5] and solved shortly afterwards independently by Wiener [6]. The LMS algorithm is a stochastic implementation of the method of the steepest descent. The LMS algorithm was introduced for adaptive noise cancellation [7]. Variations and improvements show faster convergence or better tracking abilities. A thorough examination of the LMS performance analysis was provided 
in [8]. The LMS algorithm is not demanding in computational complexity. It is relatively simple to implement. The LMS does not require measurements of the pertinent correlation functions, nor does it require matrix inversion.

A standard linear LMS filter with transversal, tapped-delay, has an output that is related to its input by the relation:

$$
y_{(n)}=\sum_{i=0}^{N-1} a_{i} x_{(n-i)}
$$

and the weights of the filter are updated using a standard LMS adaptation rule, which is derived from the Widrow-Hoff algorithm that uses an instantaneous estimation of the gradient.

$$
a(n+1)=a(n)+2 \mu \quad e(n) x(n),
$$

where $\boldsymbol{x}_{(n)}$ is the input vector, $\boldsymbol{y}_{(n)}$ is the output vector, $\boldsymbol{a}(n)$ is the $N \times 1$ tap weight coefficients vector and $\mu$ is the step-size parameter. The LMS algorithm minimises the expected value of the squared difference between the estimated output and the desired response. A more general case is to minimise $\mathrm{E}\left\{\mathrm{e}(n)^{2 N}\right\}$ [9]. This represents a general class of steepest descent algorithms for adaptive filtering which allow error minimisation in the mean fourth, sixth, ... etc. $N=1$ is the LMS and $N=2$ is the Least-Mean-Fourth (LMF). The LMF has a faster convergence than the LMS algorithm. It has generally a lower weight noise than the LMS algorithm, with the same speed of convergence. It was shown to have $3 \mathrm{~dB}$ to $10 \mathrm{~dB}$ lower Mean-Squared Error (MSE) than the LMS algorithm. There are three parameters to optimise in order to achieve the best performance: the number of tap weight coefficients of the filter, $N$, the step size parameter, $\mu$ and the number of delay elements, $\Delta$. The LMF algorithm updates the weights as follows:

$$
a_{i}(n+1)=a_{i}(n)+2 \mu_{i} \cdot e^{3}(n) \cdot x(n) .
$$

\section{VOLTERRA STRUCTURES}

In the 1950s a major work was done by Zadeh [10], Wiener et al. [11] and others that did much to clarify the nature of the problem. Gabor was the first to conceive the idea of a non-linear adaptive filter [12] and built such a filter [13]. The Volterra series is a well-known method of describing non-linear dynamic systems. It is a generalisation of the Taylor series expansion of a function. However, the Volterra series has a large number of parameters to be estimated leading to a large CPU time. The output of the filter is expressed in the form

$$
y(n)=\sum_{i_{1}=1}^{N} a_{i_{1}}^{1} x_{k-i_{1}+1}+\sum_{i_{1}=1}^{N} \sum_{i_{2}=1}^{N} a_{i_{1}, i_{2}}^{2} x_{k-i_{1}+1} x_{k-i_{2}+1}+\ldots .
$$

where $x(0), x(1), \ldots, x(N)$ are samples of the filter input. This polynomial is referred to as the Gabor-Kolomogrov polynomial or a discrete form of the Volterra series. The popularity of the Volterra filtering is due to the advancement in computer technology, which allows one to estimate the relevant Higher-Order Statistics (HOS) required to calculate the higher-order Volterra kernels for non-linear systems [14], [15]. The truncated Volterra series (the Volterra filter) is an attractive non-linear system representation because the parameters of this model are linearly related to the output.

Consider a single input single output discrete time-invariant system with non-linearities, a polynomial of order $N$ and filter length $M$. The output $Y_{k}$ is expressed in terms of the inputs $x_{k}$ as follows:

$$
\begin{aligned}
& Y_{k}=a^{0}+\sum_{i_{1}=1}^{N} a_{i_{1}}^{1} x_{k-i_{1}+1}+\sum_{i_{1}=1}^{N} \sum_{i_{2}=1}^{N} a_{i_{1}, i_{2}}^{2} x_{k-i_{1}+1} x_{k-i_{2}+1}+\ldots \\
& +\sum_{i_{1}=1}^{N} \ldots \sum_{i_{N}=1}^{N} a_{i_{1}, \ldots, i_{N}}^{N} x_{k-i_{1}+1} \ldots . x_{k-i_{N}+1}
\end{aligned}
$$

where $a_{i_{1}, \ldots, i_{n}}^{n}, i_{1}, \ldots, i_{n} \in\{1, \ldots M\}$ are referred to as the Volterra Kernels. An estimate of $x(n+1)$ can be derived via:

$$
\begin{aligned}
& \hat{x}(n+1)=a_{0}+\sum_{i_{1}=0}^{N-1} a_{i_{1}}^{1} x_{n-i_{1}}+\sum_{i_{1}=1}^{N} \sum_{i_{2}=1}^{N} a_{i_{1}, i_{2}} x\left(n-i_{1}\right) x\left(n-i_{2}\right)+\ldots . \\
& +\sum_{i_{1}=1}^{N} \ldots \sum_{i_{N}=1}^{N} a_{i_{1}, \ldots, i_{N}} x\left(n-i_{1}\right) \ldots x\left(n-i_{N}\right)
\end{aligned}
$$

where $N$ is the number of samples. A Quadratic Volterra structure consists of a parallel combination of linear and quadratic filters and has the form of:

$$
Y_{k}=a^{0}+\sum_{i_{1}=1}^{N} a_{i_{1}}^{1} x_{k-i_{1}+1}+\sum_{i_{1}=1}^{N} \sum_{i_{2}=1}^{N} a_{i_{1}, i_{2}}^{2} x_{k-i_{1}+1} x_{k-i_{2}+1}
$$

A Cubic Volterra structure has the form of:

$$
\begin{aligned}
& Y_{k}=a^{0}+\sum_{i_{1}=1}^{N} a_{i_{1}}^{1} x_{k-i_{1}+1}+\sum_{i_{1}=1}^{N} \sum_{i_{2}=1}^{N} a_{i_{1}, i_{2}}^{2} x_{k-i_{1}+1} x_{k-i_{2}+1}+ \\
& \sum_{i_{1}=1}^{N} \sum_{i_{2}=1}^{N} \sum_{i_{3}=1}^{N} a_{i_{1} i_{2}, i_{3}}^{3} x_{k-i_{1}+1} x_{k-i_{2}+1} x_{k-i_{3}+1}
\end{aligned}
$$

Adaptive LMF- Quadratic and Cubic Volterra structures were developed [16]. The LMS algorithm in the Volterra structure is replaced by an adaptive LMF algorithm. The linear, quadratic, and cubic weights of the LMF-Volterra structure are updated as:

$$
\begin{gathered}
\mathbf{a}_{i_{1}}(n+1)=\mathbf{a}_{i_{1}}(n)+2 \mu_{i_{1}} \cdot e^{3}(n) \cdot x(n) \\
\mathbf{a}_{i_{1} i_{2}}(n+1)=\mathbf{a}_{i_{1} i_{2}}(n)+2 \mu_{i_{1} i_{2}} \cdot e^{3}(n) \cdot x(n) \cdot x(n) \\
a_{i_{1} i_{2} i_{3}}(n+1)=a_{i_{1} i_{2} i_{3}}(n)+2 \mu_{i_{1} i_{2} i_{3}} \cdot e^{3}(n) \cdot x(n) \cdot x(n) \cdot x(n)
\end{gathered}
$$

The extension of the LMS-Volterra to the LMF- Volterra is done to make use of the advantages of HOS, its robustness to Gaussian noise and its tracking capability in the HOS domain [17], [18]. The parameters of the LMF algorithm 
were optimised by trial and error to achieve the best performance in terms of the speed of convergence, minimum mean-squared error and tracking. The reason for applying an LMF-based filter to the ECG signals is because it has the ability to track higher-order statistical variations while the LMS algorithm is limited to tracking variations in the second-order statistics domain only. The LMF algorithm is based on updating the error to the power four, which makes it converge faster than the LMS algorithm, which is based on updating the squared error.

\section{Model Order Selection}

The best choice of the filter order, $N$, is not generally known a priori and it is usually necessary to postulate several model orders then compute error criteria that indicate which model order to choose. One well-known criterion is the Akaike Information Criteria (AIC). The AIC determines the model order by minimising an information theoretic function

$$
\operatorname{AIC}(N)=N_{d} \ln \left(\rho_{N}\right)+2 N
$$

where $N_{d}$ is the number of data samples and $\rho_{N}$ is the estimated white noise variance, the linear prediction error variance will be used for this estimate,

$$
\rho_{m}=\sum_{t}\left|\hat{e}_{m}(t)\right|^{2} .
$$

\section{RESULTS}

Using as input fetal scalp electrode, maternal chest, and maternal transabdominal ECG signals [12], the MSE performance of the LMSQV synthesiser is compared with that of the LMFQV synthesiser. This is followed by comparing the MSE performance of the LMSCV synthesiser with that of the LMFCV synthesiser. In each case the parameters have been optimised to yield the best performance for individual signals.

\section{A. $L M S Q V$ versus $L M F Q V$ Synthesisers}

Fig. 1 shows the MSE of both the LMSQV and the LMFQV synthesisers when applied to a fetal scalp electrode ECG signal. At the start of the iterations, the MSE of the LMFQV is about $3 \mathrm{~dB}$ below that of the LMSQV. Then, both synthesisers equalise towards convergence. It is interesting to note that the fetal scalp electrode ECG signal is predominantly linear and is decomposed as follows; input $=0$ $\mathrm{dB}$, output $=-0.03 \mathrm{~dB}$ linear and $-20 \mathrm{~dB}$ quadratic.

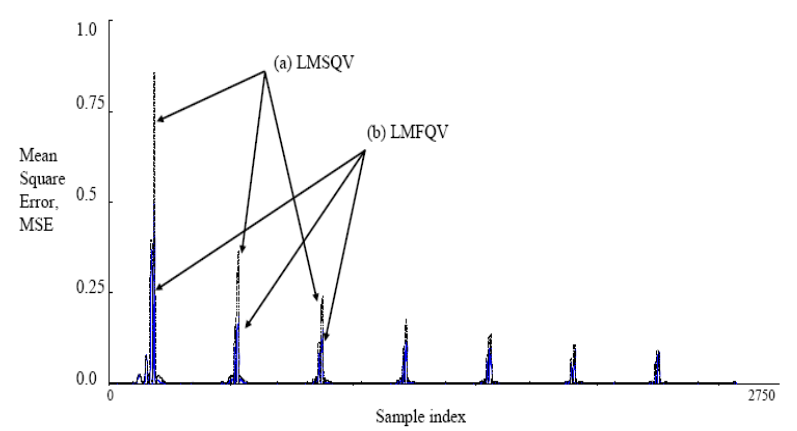

Fig. 1. The mean-squared error of (a) the LMSQV and (b) the LMFQV Synthesisers when applied to the fetal scalp electrode ECG signal.
Fig. 2 shows the MSE of both the LMSQV and LMFQV synthesisers when applied to a maternal chest ECG signal. At the start of the iterations, the MSE of the LMFQV is about 3 $\mathrm{dB}$ below that of the LMSQV. Then, both synthesisers equalise towards convergence. Note that the maternal chest ECG signal is predominantly linear and is decomposed as follows; input $=0 \mathrm{~dB}$, output $=-0.02 \mathrm{~dB}$ linear and $-19 \mathrm{~dB}$ quadratic.

Fig. 3 shows the MSE of both the LMSQV (l. h.s.) and LMFQV (r.h.s.) synthesisers when applied to a maternal transabdominal ECG signal. The LMFQV starts with an initial error which is about $39 \%$ of that of the LMSQV. The LMFQV takes only two cardiac cycles to converge whilst the LMSQV converges in nine cardiac cycles. Also, the LMFQV approaches convergence with an MSE of $2.5 \times 10^{-4}$ which is less than that of the LMSQV $\left(7.4 \times 10^{-3}\right)$; a definite improvement of $14 \mathrm{~dB}$. Note that the maternal transabdominal ECG signal is predominantly linear and is decomposed as follows; input $=0 \mathrm{~dB}$, output $=-0.015 \mathrm{~dB}$ linear and $-17 \mathrm{~dB}$ quadratic.

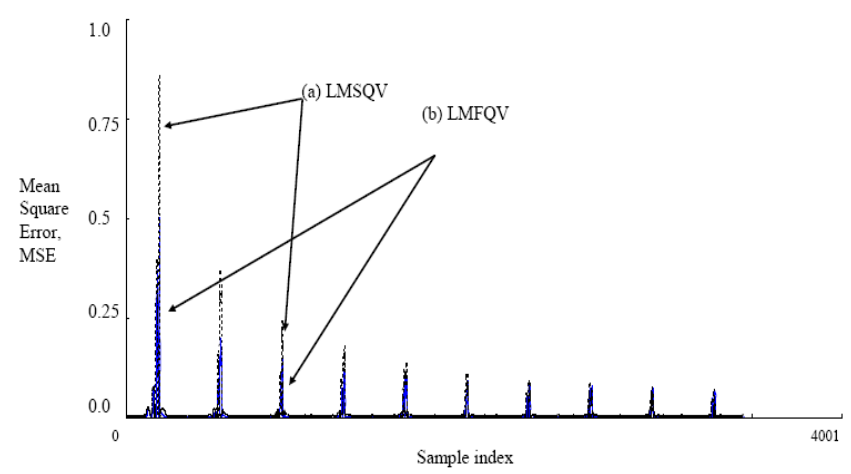

Fig. 2. The mean-squared error of (a) the LMSQV and (b) the LMFQV synthesisers when applied to the maternal chest ECG signal.

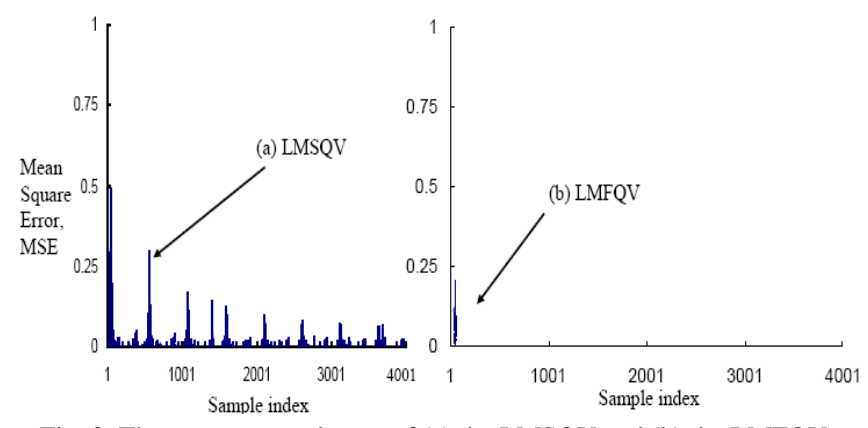

Fig. 3. The mean-squared error of (a) the LMSQV and (b) the LMFQV synthesisers when applied to the maternal transabdominal ECG signal.

\section{B. LMSCV versus LMFCV Synthesisers}

Fig. 4 shows the MSE of both the LMSCV and LMFCV synthesisers when applied to a fetal scalp electrode ECG signal. At the start of the iterations, the MSE of the LMFCV is about $3 \mathrm{~dB}$ below that of the LMSCV. Then, both synthesisers equalise towards convergence. Note that the fetal scalp electrode ECG signal is predominantly linear and is decomposed as follows; input $=0 \mathrm{~dB}$, output $=-0.03 \mathrm{~dB}$ linear, $-20 \mathrm{~dB}$ quadratic, and $-39 \mathrm{~dB}$ cubic.

Fig. 5 shows the MSE of both the LMSCV and LMFCV synthesisers when applied to a maternal chest ECG signal. At the start of the iterations, the MSE of the LMFCV is about 3 $\mathrm{dB}$ below that of the LMSCV. Then, both synthesisers 
equalise towards convergence. Note that the maternal chest ECG signal is predominantly linear and is decomposed as follows; input $=0 \mathrm{~dB}$, output $=-0.02 \mathrm{~dB}$ linear, $-19 \mathrm{~dB}$ quadratic, and $-36 \mathrm{~dB}$ cubic.

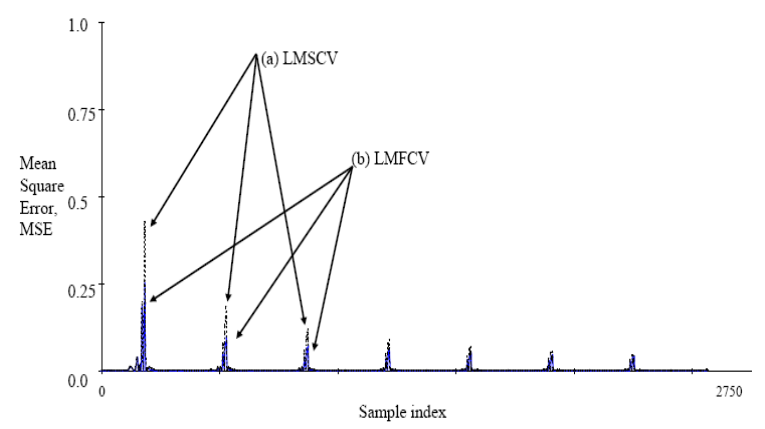

Fig. 4. The mean-squared error of (a) the LMSCV and (b) the LMFCV synthesisers when applied to the fetal scalp electrode ECG signal.

Fig. 6 shows the MSE of both the LMSCV (1. h.s.) and LMFCV (r.h.s.) synthesisers when applied to a maternal transabdominal ECG signal. At the start of iterations, the LMFCV is $6 \mathrm{~dB}$ below that of the LMSCV. The LMFCV takes only two cardiac cycles to converge whilst the LMSCV converges in nine cardiac cycles. Also, the LMFCV has a steady-state MSE of $1.5 \times 10^{-6}$ whilst that of the LMSCV is 3 $\times 10^{-5}$ which is an improvement of approximately $13 \mathrm{~dB}$. Note that the maternal transabdominal ECG signal is predominantly linear and is decomposed as follows; input $=0$ $\mathrm{dB}$, output $=-0.015 \mathrm{~dB}$ linear, $-17 \mathrm{~dB}$ quadratic, and $-33 \mathrm{~dB}$ cubic. The parameters for the adaptive LMS- and LMF-
Quadratic and Cubic Volterra synthesisers are summarised in [16] along with flowcharts and block diagrams. The parameters for the adaptive LMS- and LMF- Quadratic and Cubic Volterra synthesisers are summarised in Table I. The LMS and LMF algorithms are summarised in Table II and Table III, respectively.

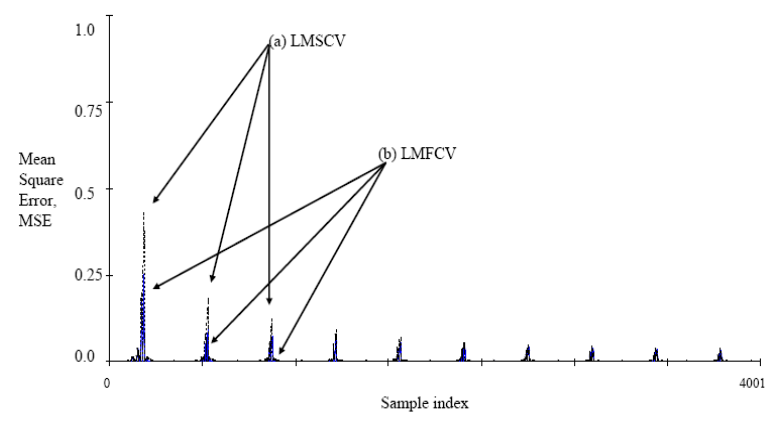

Fig. 5. The mean-squared error of (a) the LMSCV and (b) the LMFCV synthesisers when applied to the maternal chest ECG signal.

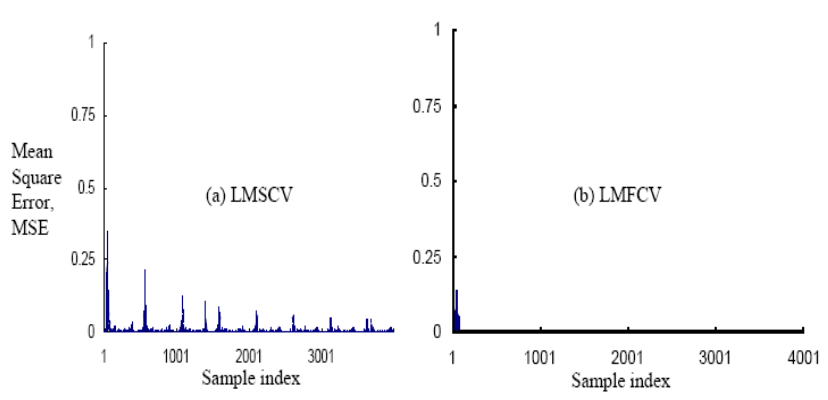

Fig. 6. The mean-squared error of (a) the LMSCV and (b) the LMFCV synthesisers when applied to the maternal transabdominal ECG signal.

TABLE I: THE OPTIMISED PARAMETERS OF THE LMSQV, LMSCV, LMFQV, AND LMFCV ADAPTIVE ALGORITHMS COMPARED IN FigS. 1-6 FOR (A) FETAL SCALP ElECtrode, (B) MATERnal Chest, AND (C) MATERnAl Transabdominal ECG Signals

\begin{tabular}{|l|c|c|l|c|}
\hline & Number of elements, $N$ & Delay, $\Delta$ & Step-size parameters, $\mu$ & CPU (msec) \\
\hline LMS Quadratic Volterra & 8 & 6 & $0.002,0.0004$ & 25 \\
\hline LMS Cubic Volterra & 6 & 6 & $0.001,0.0001,0.0001$ & 350 \\
\hline LMF Quadratic Volterra & 8 & 5 & $0.001,0.0002$ & 40 \\
\hline LMF Cubic Volterra & 6 & 5 & $0.001,0.0002,0.0004$ & 450 \\
\hline
\end{tabular}

(B)

\begin{tabular}{|l|c|c|c|c|}
\hline & Number of elements, $N$ & Delay, $\Delta$ & Step-size parameters, $\mu$ & CPU (msec) \\
\hline LMS Quadratic Volterra & 6 & 2 & $0.002,0.0004$ & 35 \\
\hline LMS Cubic Volterra & 6 & 2 & $0.001,0.0001,0.0001$ & 700 \\
\hline LMF Quadratic Volterra & 6 & 2 & $0.001,0.0002$ & 50 \\
\hline LMF Cubic Volterra & 6 & 2 & $0.001,0.0002,0.0004$ & 850 \\
\hline
\end{tabular}

(C)

\begin{tabular}{|l|c|c|c|c|}
\hline & Number of elements, $N$ & Delay, $\Delta$ & Step-size parameters, $\mu$ & CPU (msec) \\
\hline LMS Quadratic Volterra & 6 & 2 & $0.002,0.0004$ & 35 \\
\hline LMS Cubic Volterra & 6 & 2 & $0.001,0.0001,0.0001$ & 700 \\
\hline LMF Quadratic Volterra & 6 & 2 & $0.001,0.0002$ & 50 \\
\hline LMF Cubic Volterra & 6 & 2 & $0.001,0.0002,0.0004$ & \\
\hline
\end{tabular}

TABLE II: SUMMARY OF THE LMS ALGORITHM

1. Initialisation: Set $a_{i}(1)=0$

for $k=1,2, \ldots, p$

2. Filtering: For time $n=1,2, .$. compute

$y_{(n)}=\sum_{i=0}^{N-1} a_{i} x_{(n-i)}$,

$e(n)=d(n)-y(n)$,

$\mathbf{a}(n+1)=\mathbf{a}(n)+2 \mu \quad e(n) x(n)$,

for $k=1,2, \ldots, p$.
TABLE III: SUMMARY OF THE LMF ALGORITHM

1. Initialisation: Set $a_{i}(1)=0$

for $k=1,2, \ldots, p$

2. Filtering: For time $n=1,2, .$. compute

$y_{(n)}=\sum_{i=0}^{N-1} a_{i} x_{(n-i)}$,

$e(n)=d(n)-y(n)$,

$\mathbf{a}_{i}(n+1)=\mathbf{a}_{\mathrm{i}}(n)+2 \mu_{i} \cdot e^{3}(n) \cdot x(n)$.

for $k=1,2, \ldots, p$. 


\section{CONCLUSIONS}

The objective of this paper is to decompose the maternal transabdominal ECG signal into its linear, quadratic, and cubic parts and retain only the linear part. The maternal transabdominal ECG signal is a combined maternal and fetal ECG and there is also the uterine contraction interference signal (UCS) during labour combined with it. Each one of these three combined signals is non-linear by its own right. To synthesise the maternal transabdominal ECG signal, a non-linear predictor / synthesiser is sought to carry out this task because employing a linear structure to cater for such non-linear signals would lead to a suboptimal solution. The predictor / synthesiser will try to model as faithfully as possible the linear, quadratic and cubic parts of the ECG signal. When dealing with a process of a non-linear nature, the use of a Volterra structure provides a powerful method for solving the prediction problem by virtue of the non-linear processing units built into its construction. The only exception to the use of non-linear units, however, is the output of the structure, which is linearly related to its inputs. The coefficients of the Volterra filter are adjusted via gradient descent to minimise the mean squared value of the difference between the desired response and the actual filter output.

A non-linear synthesiser is sought to carry out this task. Quadratic and Cubic Volterra synthesisers are used to linearise the fetal scalp electrode, the maternal chest, and the maternal transabdominal ECG signals by removing the quadratic or the quadratic and cubic parts, respectively, and retaining only the linear part. The Volterra structures are attractive since they can deal with a general class of non-linear systems while their outputs are still linear with respect to their inputs via their linear, quadratic and cubic parts of their transfer functions.

Throughout this paper, Quadratic Volterra referred to a filter that consists of a linear and a quadratic part. A Cubic Volterra referred to a filter that consists of a linear, a quadratic, and a cubic part. Adaptive LMF- Quadratic and Cubic Volterra structures are developed and applied to full cardiac cycles of the fetal scalp electrode, the maternal chest, and the maternal transabdominal ECG signals. The extension of the conventional LMS-Volterra to the LMF-Volterra is done to make use of the advantages of higher-order statistics, especially its robustness to Gaussian noise and its tracking capability. Adaptive LMS- and LMF- Quadratic and Cubic Volterra structures are used to decompose the aforementioned ECG signals into their linear, quadratic and cubic parts and retain only the linear part. The LMFQV takes only two cardiac cycles to converge whilst the LMSQV converges in nine cardiac cycles when both structures are applied to maternal transabdominal ECG signals. Also, the LMFQV approaches convergence with an MSE which is 14 $\mathrm{dB}$ below that of the LMSQV. The LMFCV takes only two cardiac cycles to converge whilst the LMSCV converges in nine cardiac cycles when both structures are applied to maternal transabdominal ECG signals. After convergence of both synthesisers, the MSE of the LMFCV is $13 \mathrm{~dB}$ below that of the LMSCV. Hence, both the adaptive LMF-
Quadratic and Cubic Volterra structures outperform the adaptive LMS- Quadratic and Cubic Volterra structures by $14 \mathrm{~dB}$ and $13 \mathrm{~dB}$, respectively. The Cubic Volterra structure yields a better performance in terms of the MSE than the Quadratic Volterra structure by approximately $20 \mathrm{~dB}$. The CPU time could be reduced by eliminating redundant coefficients from the Cubic Volterra synthesiser [16]. In practice, however, it might be prudent to compromise and use an LMF- Quadratic Volterra as opposed to an LMF- Cubic Volterra structure. This would simplify the implementation of the algorithm in software or hardware and reduce the CPU time required by two orders of magnitude which will make it more attractive for on-line implementations in handheld or portable devices.

\section{REFERENCES}

[1] M. S. Rizk and W. A. Zgallai, "Higher order statistics are indispensable tools in the analysis of electrocardiogram signals," in Proc. IEE Colloquium on Statistical Signal Processing, January 1999.

[2] M. S. Rizk, D. Romare, W. A. Zgallai, K. T. V. Grattan, P. Hardiman and J. O'Riordan, "Higher order statistics (HOS) in signal processing: Are they of any use?" in Proc. IEE Colloquium, Digest \#111, May 1995, no. 1, pp. 1-6, London.

[3] W. A. Zgallai, "Embedded Volterra for prediction of elecro-myographic signals during labour," in Proc. The $16^{\text {th }}$ IEEE International Conference on Digital Signal Processing (DSP), Greece, July 2009.

[4] W. A. Zgallai, "Detection and classification of adult and fetal ECGusing recurrent neural networks, embedded Volterra and higher-order statistics," in Recurrent Neural Networks, M. El Hefnawi and M. Mysara, Eds. InTech Open, 2012.

[5] A. N. Kolmogorov, Interpolation and Extrapolation of Stationary Random Sequences, USA, April 1962.

[6] N. Wiener, Extrapolation, Interpolation, and Smoothing of Stationary Time Series with Engineering Applications, Cambridge, MA: MIT Press, 1949.

[7] B. Widrow et al., "Adaptive noise cancellation: principles and applications," Proceedings of IEEE, vol. 63, no. 12, pp. 1692-1716, December 1975.

[8] J. R. Zeidler, "Performance analysis of LMS adaptive prediction filters," IEEE Proceedings, vol. 78, no. 12, pp. 1781-1806, December 1990.

[9] E. Wallach and B. Widrow, "The least-mean fourth (LMF) adaptive algorithm and its family," IEEE Transactions on Information Theory, vol. IT-30, no. 2, pp. 275-283, March 1984.

[10] L. A. Zadeh, "A contribution to the theory of nonlinear systems," Journal of the Franklin Institute, vol. 255, pp. 387-401, 1953.

[11] N. Wiener, Nonlinear Problems in Random Theory, NY, Wiley, 1958.

[12] D. Gabor, "Communication theory and cybernetics," IRE Transactions on Circuit Theory, vol. CT-1, pp. 19-31, 1954.

[13] D. Gabor, W. P. L. Wilbey, and R. Woodcock, "A universal nonlinear filter, predictor and simulator which optimises itself by a learning process," Proceedings of the Institution of Electrical Engineers, London, vol. 108, pp. 422-435, 1960.

[14] S. B. Kim and E. J. Powers, "Estimation of Volterra kernels via higher-order statistical signal processing," Higher Order Statistical Signal Processing, (Boashash, Powers, and Zoubier), ch. 7, pp. 213-267, 1995.

[15] S. V. Vaseghi, Advanced Signal Processing and Digital Noise Reduction, Wiley and Teubner, 1996.

[16] W. A. Zgallai, "Advanced robust non-invasive fetal heart detection techniques during active labour using one pair of transabdominal electrodes," PhD Thesis, City University London, UK, 2007.

[17] W. A. Zgallai, "Second- and third-order statistical characterization of non-linearity and non-Gaussianity of adult and fetal ECG signals and noise," in Practical Applications in Biomedical Engineering, A. O. Andrade et al., Eds. January 9, 2013.

[18] M. S. Rizk, W. A. Zgallai, S. AlKafeef, E. R. Carson, and K. T. V. Grattan, "Higher-order ambulatory electrocardiogram identification and motion artefact suppression with adaptive second- and third-order volterra filters," SPIE '98 Advanced Signal Processing Algorithms, Architecture, and Implementations VIII, vol. 3461, pp. 417-431, USA, July 1998. 
Walid A. Zgallai was born in Tripoli in 1969. He received his BEng degree in electrical and electronic engineering from Kuwait University, Kuwait in 1992 BSc degree in electrical engineering from Tripoli University, Tripoli, Libya in 1992. He received his MSc degree in communications and signal processing from the University of London, London, UK in 1993 and a diploma of Imperial College (DIC), London, UK in 1993. He received his $\mathrm{PhD}$ degree in electrical engineering from City University London, London, UK in 2007 . He also obtained a postgraduate certificate in learning and teaching from the University of West London, London, UK in 2009.

He worked as a teaching assistant, research assistant, research associated, and postdoctoral research fellow at City University London, London, UK from 1998. He worked as a senior lecturer and the programme leader at the University of West London from 2008. He works as a faculty and the programme leader of biomedical engineering at the Faculty of Engineering
Technology and Science at the Higher Colleges of Technology, Dubai, UAE $\mathrm{He}$ authored 30 publications published in international peer-reviewed journals and conferences. His research interests include detection and classification of ECG signals including abnormalities as well as transabdominal fetal ECG signal during labour, adaptive filtering, non-linear and non-Gaussian signals in non-stationary environment, neural networks, higher-order statistics, and chaos and fractal signals.

Dr. Zgallai is a fellow of the Higher Education Academy in the UK, FHEA. He is a member of the IET, member of the IEEE, and an associate member of the Institute of Knowledge Transfer in the UK. He serves in many academic committees, including accreditations, he has been an external examiner at international $\mathrm{HE}$ institutions. 